\title{
Linx
}

Revue des linguistes de l'université Paris X Nanterre

$7 \mid 1995$

Saussure aujourd'hui

\section{»Boeuf, lac, ciel« - »concierge, chemise, lit«}

\section{Johannes Fehr}

\section{OpenEdition}

\section{Journals}

Édition électronique

URL : http://journals.openedition.org/linx/1236

DOI : 10.4000/linx.1236

ISSN : 2118-9692

\section{Éditeur}

Presses universitaires de Paris Nanterre

\section{Édition imprimée}

Date de publication : 1 septembre 1995

Pagination : 431-438

ISSN : 0246-8743

\section{Référence électronique}

Johannes Fehr, « »Boeuf, lac, ciel« - »concierge, chemise, lit« », Linx [En ligne], 7| 1995, mis en ligne le 25 juillet 2012, consulté le 20 avril 2019. URL : http://journals.openedition.org/linx/1236 ; DOI :

$10.4000 / \operatorname{linx} .1236$

Ce document a été généré automatiquement le 20 avril 2019.

Département de Sciences du langage, Université Paris Ouest 


\title{
»Boeuf, lac, ciel« - »concierge, chemise, lit«
}

\author{
Johannes Fehr
}

Pour Julia Céline

$1 \mathrm{Si}$, aujourd'hui, nous parlons d'une rencontre après coup entre Freud et Saussure, cela ne se fait, d'une manière ou d'une autre, qu'à partir et dans l'horizon de l'œuvre de Jacques Lacan. Mais autant le point de départ de ce qui sera dit ici est clair, autant il faut se demander, à mon avis, comment au juste on peut situer la dite rencontre Freud/Saussure.

2 En 1973 déjà, avec Le titre de la lettre (une lecture de Lacan) Philippe Lacoue-Labarthe et Jean-Luc Nancy ont démontré que le fameux propos de Lacan - "Freud anticipait Saussure » - ne révélait pas tant une chronologie désormais bien établie qu'il ne cachait un certain malaise de la théorie lacanienne quant à l'articulation théorique du rapport entre psychanalyse et linguistique.

3 Dans une série de travaux plus récents Michel Arrivé a mis en évidence les difficultés qu'on rencontre, si l'on veut saisir, sur un niveau strictement conceptuel, les rapports postulés par Lacan entre la psychanalyse freudienne et la linguistique saussurienne.

4 Ceci pour vous dire brièvement pourquoi, aujourd'hui, et donc bien et à plusieurs égards après coup, je tenterai d'arranger un autre genre de rencontre entre Freud et Saussure, à savoir une rencontre de textes.

5 La note manuscrite de Saussure que nous allons lire a été publiée pour la première fois par Jean Starobinski dans Les mots sous les mots en 1971 et ensuite, dans une version philologiquement plus instrumentée, par René Amacker dans les Cahiers Ferdinand de Saussure en 1989 sous le titre " Note de Ferdinand de Saussure concernant le 'discours'" . Il s'agit d'un fragment d'une vingtaine de lignes dont, à ma connaissance, la datation est incertaine sinon impossible.

6 Le texte de Freud, par contre, ne pose pas de problèmes de ce genre. Il figure dans les Etudes sur l'hystérie, que Freud a rédigées de 1891 à 1895 en collaboration avec le médecin 
viennois Joseph Breuer. Cet ouvrage, on le sait, est depuis lors devenu le premier classique de la littérature psychanalytique, traduit en français dès 1956 par les soins d'Anne Berman. L'extrait que j'ai choisi et par lequel je voudrais initier cette entrevue textuelle se trouve dans la quatrième et dernière partie du livre, signée par Freud et intitulée Psychothérapie de l'hystérie.

Une information préliminaire pour commencer. Au moment de la publication des Etudes sur l'hystérie Freud est à la recherche d'un traitement thérapeutique pour ses malades hystériques. Il ne se fie déjà plus à l'hypnose dont il avait appris et admiré la manipulation chez Charcot à la Salpêtrière et chez Bernheim à Nancy, mais il n'a pas encore découvert la dite "libre association" ou "règle fondamentale", le «simple échange de mots » qui allait devenir la clef de voûte de la technique psychanalytique. A mi-chemin entre l'une et l'autre technique Freud avait découvert quelque chose qu'il appelait «le procédé de la pression » - «Druckprozedur » - technique dont on ne parle plus guère aujourd'hui, mais qui au moment de la rédaction des Etudes sur l'hystérie lui apparaissait comme un « artifice » indispensable à sa pratique de médecin neurologiste :

Dans les cas graves d'hystérie les exhortations d'un médecin étranger, non au courant des faits, ne peuvent égaler la «résistance aux associations». Il faut recourir à des procédés plus efficaces.

Je fais, en pareil cas, usage d'un petit artifice technique. J'informe mon malade que je vais dans l'instant qui suivra, exercer une pression sur son front et lui assure que, pendant tout le temps que durera cette pression, un souvenir surgira en lui sous la forme d'une image ou bien qu'une idée se présentera à son esprit. [...]

Ce procédé, tout en me permettant d'arriver au but visé, m'a appris bien des choses ; je ne saurais plus m'en passer aujourd'hui. » (ESH, p. 217/18)

8 C'est de ce "procédé de pression » ainsi préconisé par Freud qu'il est question dans le passage qui nous intéresse plus particulièrement:

Il arrive parfois que les renseignements obtenus par le procédé de la pression se produisent sous une forme très curieuse [...]. Je me souviens ainsi d'une dame souffrant depuis de nombreuses années d'idées obsédantes et de phobies qui, tout en rapportant l'apparition de ses troubles à ses années d'enfance, en ignorait absolument la cause. Sincère et intelligente, elle ne m'opposait qu'une résistance consciente à peine perceptible. [...]

Quand je demandai à cette dame si, sous la pression de ma main, elle avait vu quelque chose, elle me répondit : «Rien du tout, mais tout à coup j'ai pensé à un mot.» - «A un seul mot?» - « Oui, mais ça me semble trop bête.» - «Dites-le quand même.» - « Eh bien, concierge.» - «Rien d'autre?» - « Non. » J'exerce une deuxième pression et un autre mot lui traverse l'esprit: "Chemise." - Je remarque alors qu'il y a ici une nouvelle façon de répondre et, en répétant la pression, je provoque l'énonciation d'une série de mots en apparence dénuée de sens : «Concierge, chemise, lit, ville, charette.» - «Que signifie tout cela?» (ESH, p. 222)

Arrêtons-nous ici pour l'instant et passons tout de suite au texte de Saussure. Dans sa note concernant le 'discours' il n'est point question d'hystérie ni de «dame sincère et intelligente ", mais, plus sobrement en apparence et avec des phrases pleines d'hésitations et de ratures, du rapport entre le concept de «langue» et celui de « discours »:

La langue n'est créée qu'en vue du discours, mais qu'est-ce qui sépare le discours de la langue, ou qu'est-ce qui, à un certain moment, permet de dire que la langue entre en action comme discours?

Des concepts variés sont là, prêts dans la langue, (c'est-à-dire revêtus d'une forme \{linguistique\} tels que boeuf, lac, ciel, fort, rouge, triste, cinq, fendre, voir. A quel 
moment ou en vertu de quelle opération, de quel jeu \{qui s'établit entre eux\}, \{de quelles conditions\}, ces concepts formeront-ils le DISCOURS ?

La suite de ces mots, si riche qu'elle soit, n'indiquera jamais par les idées qu'elle évo que, n'indiquera jamais à un individu humain qu'un autre individu, en les prononçant, veuille lui signifier quelque chose. Que faut il pour p que nous ayons l'idée qu'on veut $X X X$ signifier quelque chose, en usant des innombrables termes qui sont à disposition dans la langue ? (CFS 43, p. 94)

10 Je me permets d'interrompre la lecture de la note saussurienne, comme je l'ai déjà fait avec la citation de Freud, proprement sur le point d'interrogation, en laissant provisoirement en suspens les réponses respectives qu'ils donneront. Car c'est à partir des questions qu'ils relèvent que l'intrication des problèmes auxquels Freud et Saussure se heurtaient l'un et l'autre pourra être révélée.

11 Commençons avec la question de Saussure : "Que faut il pour p que nous ayons l'idée qu'on veut $X X X$ signifier quelque chose, en usant des innombrables termes qui sont à disposition dans la langue? »Cette question vise un problème crucial de la théorie saussurienne, à savoir le rapport entre le système, « la langue », et les sujets parlants. La théorie saussurienne, vous le savez, se constitue précisément, et pour des raisons bien évidentes, en définissant « la langue » comme le seul objet scientifiquement valable d'une linguistique à venir. Et cet objet, « la langue », est caractérisé comme " un système qui ne connaît que son ordre propre ». (CLG/D, p.Ê43) Mais, si « dans le système de la langue il n'y a que des différences" (CLG/D, p.Ê166), la question se pose, en effet, de savoir comment les sujets parlants peuvent s'en servir pour "signifier quelque chose ». Cette question, bien qu'elle ne soit apparemment pas développée dans le CLG, n'est point étrangère aux préoccupations théoriques de Saussure, comme le montrent ces quelques phrases de ses notes dites «Item » :

Item. Il y a défaut d'analogie entre la langue et toute autre chose humaine pour deux raisons: $\left[1^{\circ}\right]$ La nullité interne des signes. $-\left[2^{\circ}\right]$ La faculté de notre esprit de s'attacher à un terme en soi nul. - (CLG/E (II), p. 38, N 15, nº 3316.1)

Et comme pour confirmer ce qu'il vient d'écrire, Saussure ajoute entre parenthèses : (Mais ce n'était pas ce que je voulais dire d'abord. J'ai dévié.) (ibid.)

Ecoutons maintenant la réponse que Saussure donne à la question posée dans sa note sur le 'discours' :

Que faut il pour $p$ que nous ayons l'idée qu'on veut $X X X$ signifier quelque chose, en usant des innombrables termes qui sont à disposition dans la langue ? C'est la même question que de savoir ce qu'est le discours, par opposition à la simple notion de langue et à première vue la réponse est simple: le discours consiste, ffût-ce rudimentairement, et par des voies que nous ignorons\}, à affirmer un lien entre deux des concepts qui se présentent revêtus \{préalablement\} d 'une e la forme linguistique, pendant que la langue ne fait \{préalablement\} que réaliser des concepts isolés, qui attendent d'être mis en rapport entre eux pour qu'il y ait signification de pensée. (CFS 43, p. 94)

12 «La réponse est simple "- mais seulement "à première vue ». Car, si « le discours consiste à affirmer un lien entre deux concepts ", c'est justement, comme Saussure l'avoue explicitement, "par des voies que nous ignorons ». Manière de dire que la réponse ne saurait être simple ou qu'elle est simple dans ce sens qu'elle renvoie, simplement, à une autre question qui, elle, restera sans réponse dans ce fragment. Or, précisément, comment un lien entre deux concepts peut-il s'affirmer?

13 Si Saussure avoue l'ignorer, il ne peut s'agir d'un lien syntaxique, voire logique, du type «sujet-prédicat » tel que la grammaire le décrit depuis des millénaires. La question de 
Saussure vise donc ailleurs - dans la direction peut-être de cet autre fragment de ses notes «Item » :

Item. La réalité de l'existence des fils qui relient entre eux les éléments d'une langue, - bien que fait psychologique immense, - n'a pour ainsi dire pas besoin d'être démontrée. C'est cela même qui fait la langue. (CLG/E (II), p.36, N 15, n - 3310.4)

«La réalité de l'existence des fils », «bien que fait psychologique immense », n'aurait - je répète - "pour ainsi direpas besoin d'être démontrée ». Ne faut-il pas plutôt entendre ici que c'est justement cette "réalité »-là - « cela même qui fait la langue » - qui reste et qui résiste à « être démontrée » ?

Revenons à Freud. Avec "le procédé de la pression» il a provoqué chez sa patiente «l'énonciation d'une série de mots en apparence dénuée de sens » : «Concierge, chemise, lit, ville, charette.»

Que signifie tout cela?» Elle réfléchit un moment puis une idée lui vient à l'esprit : «Il ne peut s'agir que d'une histoire dont je me souviens maintenant. J'avais 10 ans et celle de mes soeurs dont l'âge se rapprochait le plus du mien - elle avait 12 ans eut, une nuit, un accès de délire. On fut obligé de la ligoter et de la transporter sur une charette à la ville. Je sais avec certitude que c'est le concierge qui la maîtrisa et qui la conduisit ensuite à la maison de santé." Nous persévérons dans notre méthode de recherche et notre oracle nous fait entendre d'autres paroles que nous ne pûmes toutes interpréter, mais qui permirent de poursuivre cette histoire et de lui en rattacher une autre. Bientôt aussi nous arrivâmes à saisir la signifcation de ces réminiscences. (EHS, p. 222/23)

Chez Freud, donc, " un lien s'affirme ». La " série de mots en apparence dénuée de sens » renvoie à une histoire, et à cette histoire se rattache une autre qui mènera à «la signification de ces réminiscences». Les "voies» sur lesquelles, dans la pratique de Freud, « un lien s'affirme », c'est l'histoire ignorée du sujet parlant, ce sont les traces d'événements douloureux, oubliés ou refoulés, qui déterminent le sujet parlant dans ce qu'il dit. Or, il est clair que les "voies » ainsi découvertes par Freud et sa patiente ont quelque chose de déroutant pour le linguiste. En suivant la lettre du CLG il faut même dire d'emblée que "l'oracle» psychanalytique ne saurait être entendu puisqu'il mène directement dans la sphère de l'individu ou de l'individuel qu'il s'agissait précisément d'éviter avec la théorie de «la langue ». Mais cela n'empêche pas que dans la note sur le 'discours' que nous venons de parcourir, quelque chose insiste : ce que l'on peut deviner, à travers les hésitations mêmes du fragment saussurien, c'est que le système de «la langue » - " pour entrer en action comme discours » - présuppose, de par son intérieur, un rapport nécessaire à une instance qui doit rester extérieure et étrangère à son « ordre propre », mais qui fait « lien» entre les concepts et qu'on pourrait nommer, avec Freud, l'histoire du sujet parlant.

Mais dans cette rencontre de textes ce n'est pas seulement celui du psychanalyste qui peut donner une interprétation aux lignes de la main du linguiste. Car si Freud, avec l'histoire refoulée du sujet, trouve une «signification » aux « séries de mots en apparence dénuées de sens » et dispose dès lors d'un outil thérapeutique efficace, la provenancede ces "séries de mots", la possiblilité de pouvoir les produire ou les "provoquer" par «le procédé de la pression » lui pose un grave problème théorique.

En effet, comment s'expliquer que des patientes, qu'elles soient «sincères et intelligentes » ou non, puissent énoncer, « sous la pression de la main», des « séries de 
mots » qui renvoient à des histoires qu'elles ignorent "absolument»? Voici comment Freud, dans les Etudes sur l'Hystérie, essaie de trouver la solution de cette énigme :

Tous les résultats de ce procédé donnent l'impression trompeuse qu'il existe, en dehors du conscient des sujets, une intelligence supérieure qui détient et groupe dans un but déterminé d'importants matériaux psychiques. Elle semble avoir trouvé, pour le retour dans le conscient de ceux-ci, un ingénieux arrangement. Mais je suppose que cette seconde intelligence inconsciente n'est qu'apparente. (EHS, p. 219)

19 Cette « seconde intelligence supérieure et inconsciente » dont Freud parle ici avec tant de précaution en nous avertissant qu'il se pourrait bien qu'elle ne soit qu'une «impression trompeuse » ou seulement « apparente » sera bientôt appelée « l'inconscient » - en un seul mot.

20 Mais, ici encore, ce qui précède l'état doctrinal de la théorie me semble comporter un moment d'ouverture ou d'hésitation qui rend lisible des questions qu'il sera plus difficile de poser après coup. Car, si le fameux «inconscient» doit être considéré à bon droit comme pierre angulaire de l'édifice freudien, cela ne veut pas dire pour autant qu'il soit clair, même et peut-être surtout dans les textes de Freud, comment il faut le concevoir et où le situer.

21 Il faut prêter l'oreille «à la façon imagée de s'exprimer » (ESH, p. 233), aux tournures tordues auxquelles Freud se voit obligé de recourir là où, justement, le mot juste lui manque. C'est « une série de comparaisons n'ayant qu'une très vague ressemblance avec le sujet dont je m'occupe » (ESH, p. 235) nous dit Freud, mais explique-t-il : «c'est afin de rendre compréhensibles, par divers rapprochements, des idées extrêmement compliquées et jamais encore exposées. » (Ibid.)

Ecoutons : il s'agirait donc d' " une intelligence supérieure - en dehors de la conscience du sujet - qui détient et groupe dans un but déterminé d'importants matériaux psychiques. Elle semble avoir trouvé, pour le retour dans le conscient de ceux-ci, un ingénieux arrangement. » Plus loin dans le texte Freud parlera aussi d'une « disposition ayant un caractère dynamique » (ESH, p. 234), parlera de « séries » (p. 233) ou encore d'un « système de lignes ramifiées » et de « noeuds » (p. 234).

23 Vous l'aurez remarqué: On rencontre, littéralement, les mêmes mots et de pareils « rapprochements » dans les notes où Saussure essaie de saisir l'objet fuyant dont il avait besoin pour le fondement de la linguistique. Plutôt qu'à aucune réalité biologique, «l'intelligence supérieure en dehors du conscient des sujets» que Freud découvre à travers les « séries de mots en apparence dénuées de sens » fait donc penser au système de « la langue » tel que Saussure l'esquissa.

24 Or, quelles conclusions faut-il tirer de cette rencontre de textes?

25 Je dirais d'abord qu'on peut ré-affirmer, à la suite de Lacan, qu'il y a un enjeu théorique véritable entre psychanalyse et linguistique, ou en d'autres termes: il me parait qu'une lecture d'inspiration psychanalytique des textes de Saussure reste toujours à faire.

Ce qui rapproche Freud et Saussure, notamment, ce sont les nombreux passages où ils se consacrent, l'un et l'autre, à l'analyse de ce que j'appellerai des complexes discursifs, c'està-dire des syntagmes de différente longueur qui ne sont pas réductibles à des relations purement syntaxiques: chaînes et traits de mots, lapsus etc. du côté de chez Freud, anagrammes, légendes, productions glossolaliques et autres chez Saussure. C'est la même question qui hante tout ces textes : la question de savoir - qui parle? 
27 Mais cela ne veut pas dire pour autant que la linguistique saussurienne et la psychanalyse freudienne puissent être subsumées sous un même savoir positif. Car plutôt que de se compléter, les textes que nous venons de lire renvoient l'un à l'autre, renvoient à ce qui, dans l'autre, se dérobe.

\section{BIBLIOGRAPHIE}

FREUD Sigmund et Joseph Breuer, 1956. Etudes sur l'hystérie, traduit de l'allemand par Anne Berman, PUF, Paris [EHS].

SAUSSURE Ferdinand de, 1972. Cours de linguistique générale, édition critique préparée par Tullio De Mauro, Payot, Paris [CLG/D].

SAUSSURE Ferdinand de, 1974. Cours de linguistique générale, édition critique par Rudolf Engler, tome 2, fascicule 4, Notes de F. de Saussure sur la linguistique générale, Otto Harassowitz, Wiesbaden [CLG/E (II)].

SAUSSURE Ferdinand de, 1989. « Note concernant le 'discours'", publiée par René Amacker, in : Cahiers Ferdinand de Saussure43, p. 93-94 [CFS 43]. 